\title{
Higiene Oral y Salud Periodontal en Trabajadores Agrícolas Migrantes y su Asociación con el Alfabetismo Funcional en Salud Oral
}

\author{
Oral Hygiene and Periodontal Health in Migrant Farm Workers \\ and its Association with Functional Literacy in Oral Health
}

\author{
Maricela García Domínguez; Tamara Otzen²; Javier de la Fuente-Hernández \& Carlos Manterola²
}

GARCÍA, M. D.; OTZEN, T.; DE LA FUENTE-HERNÁNDEZ, J. \& MANTEROLA, C. Higiene oral y salud periodontal en trabajadores agrícolas migrantes y su asociación con el alfabetismo funcional en salud oral. Int. J. Odontostomat., 15(1):159$165,2021$.

RESUMEN: En México las enfermedades orales se encuentran entre las de mayor demanda de atención en los servicios de salud, presentándose en las poblaciones de trabajadores migrantes mayor vulnerabilidad en cuestiones de salud. El alfabetismo funcional en salud oral es la capacidad que los individuos poseen para obtener, procesar y comprender la información de los servicios de salud básica, reflejándose en su salud. El objetivo de este estudio fue determinar el estado de higiene oral y su relación con el nivel de alfabetismo funcional (OHLA-S) en salud oral de los trabajadores agrícolas migrantes mexicanos. Estudio de corte transversal, con un tamaño de muestra de 208 sujetos elegidos por conveniencia y calculada con base a un OR esperado. Se incluyeron a sujetos de 18 años o más, con consentimiento informado. Las variables estudiadas fueron: autocuidado, OHLA-S, índice de higiene oral simplificado (IHO-S) e índice periodontal comunitario (IPC). Se aplicó estadística descriptiva y analítica. El 53,0 \% de la muestra fueron hombres. La media de edad fue de $30,7 \pm 10,8$ años. Los promedios de alfabetismo funcional en salud oral, del índice de higiene y del índice periodontal comunitario fueron de $7,8 \pm 6,9 ; 1,7 \pm 1,0 ;$ y 2,0 0 , 7 respectivamente. La regresión lineal múltiple del IPC muestra que edad y puntaje del OHLA-S explican el 22,0\% de la varianza y el OHLA-S influye indirectamente en el IHO-S. El promedio del índice de higiene oral de los trabajadores agrícolas migrantes fue bueno y el promedio del índice periodontal comunitario reflejó presencia de gingivitis. Así mismo, presentaron un puntaje bajo de alfabetismo funcional en salud oral; Sin embargo el alfabetismo funcional en salud oral se relacionó de forma significativa con el estado periodontal.

PALABRAS CLAVE: migrantes, trabajador, jornalero, estado de salud oral, alfabetismo funcional en salud oral, alfabetismo en salud oral, higiene oral, periodontal.

\section{INTRODUCCIÓN}

El alfabetismo funcional en salud oral es la capacidad que las personas poseen para obtener, procesar y comprender información básica proporcionada por las instituciones y profesionales de la salud. Este constructo, es influenciado por factores culturales y de conocimientos conceptuales (Burgette et al., 2016) constituyendo en la actualidad un buen indicador para mejorar la capacidad de adquisición de nuevos conocimientos así como de generación de estra- tegias frente a las demandas de salud (Kanupuru et al., 2015); porque motiva la capacidad de entender y utilizar la información en pro de mantener un buen estado de salud y conservarla (Lee et al., 2015). Así, en el aspecto asistencial, se ha reportado que las personas con un nivel alto de alfabetismo tienen mejor conocimiento de las funciones que lleva a cabo el equipo de profesionales en el consultorio (Baskaradoss, 2016) así como una menor tendencia a faltar a las

\footnotetext{
1 Departamento de Salud Pública y Odontología Comunitaria Escuela Nacional de Estudios Superiores, Unidad León. Universidad Nacional Autónoma de México.

${ }^{2}$ Centro de Excelencia en Estudios Morfológicos y Quirúrgicos (CEMyQ), Universidad de La Frontera, Chile.
} 
GARCÍA, M. D.; OTZEN, T.; DE LA FUENTE-HERNÁNDEZ, J. \& MANTEROLA, C. Higiene oral y salud periodontal en trabajadores agrícolas migrantes y su asociación con el alfabetismo funcional en salud oral. Int. J. Odontostomat., 15(1):159-165, 2021.

citas durante el tratamiento dental (Pippi et al., 2017). Además, también se ha estudiado el impacto de esta variable directamente en la salud oral, observándose una relación entre el nivel de alfabetismo funcional y la experiencia de caries, higiene oral y otras condiciones (Haridas et al., 2014; Blizniuk et al., 2015).

El alfabetismo en salud oral puede verse influenciado por factores de tipo sociodemográficos, como los económicos, educativos y de migración; respecto a esta última, se conoce que las poblaciones migrantes son grupos de personas que poseen mayor vulnerabilidad en cuestiones de salud (Lee et al., 2015).

México presenta un fenómeno de migración interna en el que las personas que pertenecen a regiones rurales de extrema pobreza se desplazan de manera temporal hacia regiones agrícolas de exportación (Juárez-Sánchez, 2015). Está migración es realizada por trabajadores eventuales, responsables de la siembra, recolección y cuidado de diversos cultivos en México. Los migrantes laboran en diferentes lapsos al año en una región agrícola y al término del ciclo agrícola se desplazan a otra región en producción o retornan a sus lugares de origen (SEDESOL, 2016). De acuerdo a la Encuesta Nacional de Jornaleros Agrícolas (Encuesta Nacional de Jornaleros Agrícolas, 2009) en México existen 9 millones 206 mil 429 jornaleros agrícolas (Gamboa Montejano \& Gutiérrez Sánchez, 2015), sin embargo no se conoce cuántos de ellos son migrantes. En México no se tienen reportes del nivel de alfabetismo funcional en salud oral, ni tampoco del estado de salud oral de los jornaleros agrícolas; Sin embargo, se ha reportado que para los migrantes, el alfabetismo está relacionado con sus creencias sobre la salud, afectando la manera en que se comportan ante las indicaciones de los profesionales de la salud (Lee et al., 2015). Por otra parte se ha reportado que ciertos factores socioeconómicos y sociodemográficos están asociados con la higiene oral, por ejemplo se ha reportado que las personas que viven en áreas urbanas y con un nivel bajo de escolaridad presentan mala higiene (Ospina et al., 2016).

El reporte de este manuscrito se realizó de acuerdo con la lista de verificación para estudios observacionales descriptivos MInCir (Manterola \& Astudillo, 2013).

El objetivo de este estudio fue determinar el estado de higiene oral y su relación con el nivel de alfabetismo funcional (OHLA-S) en salud oral de los trabajadores agrícolas migrantes mexicanos.

\section{MATERIAL Y MÉTODO}

Diseño: Estudio de corte transversal.

Participantes: Trabajadores agrícolas migrantes que llegaron a la Ciudad de Yurécuaro, Michoacán en México y se hospedaron en el albergue del SEDESOL en los meses de mayo, octubre y noviembre de 2016.

Criterios de inclusión: Se incluyeron a los sujetos que cumplieran con los siguientes criterios: ser trabajadores agrícolas migrantes de 18 años o más, que accedieron a participar en el estudio y firmaron el consentimiento informado. Se excluyeron a aquellos trabajadores con alguna discapacidad física o mental que les impidiera responder la encuesta.

Técnica de muestreo: Se utilizó un muestreo noprobabilístico por conveniencia, entrevistando a todas las personas disponibles en el albergue hasta cumplir con el tamaño mínimo.

Tamaño de la muestra: Se estimó con base a un OR de 2,5 y un valor de alfa de 0,05.

Variables: Las variables dependientes fue el índice de higiene oral simplificado (IHO-S) y el índice periodontal comunitario (IPC). Las variables independientes fueron edad, sexo, ingreso económico, años de escolaridad, visitas al dentista, seguro de salud y alfabetismo funcional en salud oral.

Protocolo de medición y recolección de datos: $\mathrm{La}$ recolección de datos fue ejecutada por una odontóloga previamente entrenada en la aplicación de los índices IHO-S e IPC (Kappa de 0,80). El examen oral se llevó a cabo siguiendo todas las recomendaciones de la Organización Mundial de la Salud, con el sujeto recostado y utilizando luz artificial. Posteriormente, se obtuvieron datos sociodemográficos y se aplicó el instrumento de alfabetismo funcional en salud oral (Oral Health Literacy AssessmentSpanish) (OHLA-S) (Lee et al., 2013). Para la aplicación de este instrumento, se le entregó a cada participante una ficha con tres columnas: la primera contenía 30 términos odontológicos, los que debían ser leídos en voz alta por cada uno de los entrevistados. La siguiente, estaba compuesta por 30 palabras relacionadas con el término odontológico; y la tercera tenía 30 palabras confusoras. Los participantes tenían que elegir entre estas últimas dos columnas la palabra que relacionaban con aquella que habían 
pronunciado (Tabla I). Se instruyó a los participantes sobre no relacionar las palabras que no conocían con el fin de disminuir la asociación por casualidad. Por su parte, la investigadora tenía otro instrumento con el nombre del paciente, en el cual marcó los aciertos y errores detectados.

Estadísticas: Los datos fueron introducidos a una base de datos y posteriormente analizados en el programa estadístico SPSS 13.0. Se calcularon medidas de tendencia central y frecuencias. Los puntajes del OHLA-S, IHO-S e IPC fueron correlacionados con las variables demográficas continuas utilizando $r$ de Pearson, además se contrastaron promedios con las variables demográficas discretas, utilizando $t$ de student para muestras independientes. También se identificó la asociación entre los puntajes de OHLA$\mathrm{S}$, IHO-S e IPC, usando la correlación de Pearson. Por último, se utilizaron regresiones lineales simples y múltiples para determinar los efectos en los puntajes de OHLA-S, considerando este como variable dependiente y todas las variables independientes del estudio. Se consideró como estadísticamente significativo un valor de $p$ de 0,05 .

Tabla I. Instrumento de Alfabetismo funcional en Salud oral: Oral Health Literacy Spanish.

\begin{tabular}{clll}
\hline 1 & Azúcar & Dulce & Amargo \\
2 & Fumar & Pulmón & Estomago \\
3 & Hilo dental & Limpio & Enjuague \\
4 & Cepillar & Pasta de dientes & Jabón \\
5 & Pulpa & Nervio & Lengua \\
6 & Flúor & Proteger & Destruir \\
7 & Frenos & Alinear & Torcer \\
8 & Genética & Familia & Amigo \\
9 & Restauración & Tratamiento & Instrumento \\
10 & Bruxismo & Rechinar & Tragar \\
11 & Absceso & Pus & Mucosidad \\
12 & Extracción & Sacar & Reemplazar \\
13 & Dentadura postiza & Sintético & Natural \\
14 & Esmalte & Superficie & Adentro \\
15 & Dentición & Dientes & Boca \\
16 & Calculo & Duro & Suave \\
17 & Encía & Rosada & Blanca \\
18 & Mal oclusión & Mordida & Herida \\
19 & Incipiente & Temprano & Tarde \\
20 & Caries & Cavidad & Ulcera \\
21 & Periodontal & Encías & Paladar \\
22 & Sellador & Cubrir & Taladrar \\
23 & Hipoplasia & Defecto & Intacto \\
24 & Halitosis & Aliento & Tos \\
25 & Analgésico & Aspirina & Vitamina \\
26 & Celulitis & Infección & Hemorragia \\
27 & Fistula & Drenaje & Verruga \\
28 & Temporomandibular & Articulación & Cuello \\
29 & Hiperemia & Sangre & Saliva \\
30 & Apicectomía & Raíz & Corona \\
\hline & & & \\
\hline
\end{tabular}

Principios éticos: Se obtuvo la aprobación del proyecto por parte del Comité de Ética de la Escuela Nacional de Estudios Superiores (ENES), Unidad León, de la Universidad Nacional Autónoma de México, se diseñó el consentimiento informado, se borraron los nombres de la base de datos al ser analizadas y se mantuvo en anonimato la participación de cada uno de los sujetos.

Financiamiento: No hubo fuentes formales de financiamiento.

\section{RESULTADOS}

De un total de 208 individuos incluidos en el estudio, el 53,0 \% son hombres, con una media de edad de $31,0 \pm 11,0$ años. Así mismo se observó que el $26,4 \%$ de los participantes reportaron tener educación formal y el 90,4 \% percibieron un ingreso de tres salarios mínimos al día. El puntaje promedio del nivel de alfabetismo en la población fue de $8,0 \pm 6,8$. El análisis de OHLA-S por variables sociodemográficas mostró una media de 7,8 66,9 en mujeres y $8,0 \pm 6,8$ en hombres $(p=0,6))$. También se observó que las personas con educación formal presentaron en promedio, mayor puntaje del OHLA-S $(10,3 \pm 6,3$, comparado con quienes no tuvieron $(p<0,001)$. Además, se observó un mayor promedio de OHLA-S en las personas con mayor ingreso económico $(8,3 \pm 6,9, p=0,02)$, sin embargo el visitar o no al dentista no presentó diferencias en los promedios de OHLA-S (Tabla II). El promedio de IHO-S que obtuvo la población fue de $1,7 \pm 0,7$, indicando que los trabajadores tienen un grado regular de higiene, sin embargo, no se presentaron diferencias estadísticamente significativas por variables sociodemográficas ni por visitar al dentista. En relación al IPC, la población obtuvo un promedio de $2,0 \pm 0,3$, indicando mayormente la presencia de cálculo supra e infragingival, sin embargo, no se presentaron diferencias estadísticamente significativas por variables sociodemográficas, ni visitar al dentista (Tabla III). 
GARCÍA, M. D.; OTZEN, T.; DE LA FUENTE-HERNÁNDEZ, J. \& MANTEROLA, C. Higiene oral y salud periodontal en trabajadores agrícolas migrantes y su asociación con el alfabetismo funcional en salud oral. Int. J. Odontostomat., 15(1):159-165, 2021.

Tabla II. Relación del OHLA-S por variables sociodemográficas y visitar al dentista.

\begin{tabular}{|c|c|c|c|c|c|}
\hline & Total & $\begin{array}{c}\text { Media }( \pm \mathrm{DE}) \\
7,8 \pm 6,9\end{array}$ & $\begin{array}{l}t \\
-\end{array}$ & $\begin{array}{l}p \\
-\end{array}$ & $\begin{array}{c}95 \% \mathrm{Cl} \\
-\end{array}$ \\
\hline \multirow[t]{2}{*}{ Sexo } & Hombres & $8,0 \pm 6,8$ & 0,472 & 0,638 & $-2,354 / 1,445$ \\
\hline & Mujeres & $7,6 \pm 7,1$ & & & \\
\hline \multirow[t]{2}{*}{ Escolaridad } & Con escolaridad & $10,3 \pm 6,3$ & 16.101 & $<0,001$ & $-10,659 /-8,334$ \\
\hline & Sin escolaridad & $0,8 \pm 2,2$ & & & \\
\hline Ingreso económico & $\begin{array}{l}<3 \text { salarios mínimos } \\
>3 \text { salarios mínimos }\end{array}$ & $\begin{array}{l}3,1 \pm 5,4 \\
8,3 \pm 6,9\end{array}$ & 3,983 & 0,022 & $-7,912 /-2,532$ \\
\hline Seguro de Salud & $\begin{array}{l}\mathrm{Si} \\
\mathrm{No}\end{array}$ & $\begin{array}{l}7,82 \pm 7,1 \\
7,81 \pm 6,8\end{array}$ & 0,007 & 0.947 & $-2,102 / 2,086$ \\
\hline \multirow[t]{2}{*}{ Visita al dentista } & $\mathrm{Si}$ & $9,1 \pm 6,9$ & 2,415 & 0,989 & $0,4233 / 4,187$ \\
\hline & $\begin{array}{l}\text { No } \\
\text { No }\end{array}$ & $\begin{array}{l}6,8 \pm 6,7 \\
7,8 \pm 6,8\end{array}$ & & & \\
\hline
\end{tabular}

Tabla III. Relación IHO-S e IPC por variables sociodemográficas y visita al dentista.

\begin{tabular}{|c|c|c|c|c|c|c|c|c|c|}
\hline & \multicolumn{4}{|c|}{$\mathrm{IHO}-\mathrm{S}$} & \multicolumn{4}{|c|}{ IPC } \\
\hline & & Media $( \pm D E)$ & $t$ & $p$ & $95 \% \mathrm{Cl}$ & Media( $\pm D E)$ & $t$ & $p$ & $95 \% \mathrm{Cl}$ \\
\hline \multicolumn{2}{|c|}{ Total } & $1,7 \pm 0,6$ & - & - & - & $2,0 \pm 0,7$ & - & - & - \\
\hline Sexo & $\begin{array}{l}\text { Hombres } \\
\text { Muieres }\end{array}$ & $1,2 \pm .0,6$ & 1,527 & 0,748 & $-0,486 / 0,061$ & $2,0 \pm 0,7$ & 0,840 & 0,363 & $-0,284 / 0,114$ \\
\hline \multirow[t]{2}{*}{ Escolaridad } & $\begin{array}{l}\text { Mujeres } \\
\text { Con } \\
\text { escolaridad }\end{array}$ & $\begin{array}{l}1,0 \pm 0,6 \\
1,1 \pm 0,6\end{array}$ & 1,018 & 0,959 & $-0,150 / 0,472$ & $\begin{array}{l}1,9 \pm 0,6 \\
1,9 \pm 0,7\end{array}$ & 3,900 & 0,053 & $0,213 / 0,649$ \\
\hline & $\begin{array}{l}\text { Sin } \\
\text { escolaridad }\end{array}$ & $1,1 \pm 0,6$ & & & & $2,3 \pm 0,6$ & & & \\
\hline \multirow[t]{2}{*}{$\begin{array}{l}\text { Ingreso } \\
\text { económico }\end{array}$} & $\begin{array}{l}<3 \text { salarios } \\
\text { mínimos }\end{array}$ & $1,0 \pm 0,6$ & 0,316 & 0,722 & $-0,541 / 0,392$ & $2,2 \pm 0,6$ & 1,540 & 0,765 & $-0,073 / 0,598$ \\
\hline & $\begin{array}{l}>3 \text { salarios } \\
\text { mínimos }\end{array}$ & $1,1 \pm 0,6$ & & & & $1,9 \pm 0,7$ & & & \\
\hline $\begin{array}{l}\text { Seguro de } \\
\text { Salud }\end{array}$ & $\begin{array}{l}\mathrm{Si} \\
\text { No }\end{array}$ & $\begin{array}{l}1,8 \pm 1,0 \\
1,5 \pm 1,0\end{array}$ & 1,360 & 0,799 & $-0,511 / 0,093$ & $\begin{array}{l}2,0 \pm 0,7 \\
1,9 \pm 0,7\end{array}$ & 0,461 & 0,632 & $-0,271 / 0,168$ \\
\hline $\begin{array}{l}\text { Visita al } \\
\text { dentista }\end{array}$ & $\begin{array}{l}\text { Si } \\
\text { No }\end{array}$ & $\begin{array}{l}1,6 \pm 0,9 \\
1,8 \pm 1,0\end{array}$ & 1,243 & 0,800 & $-0,449 / 0,102$ & $\begin{array}{l}2,1 \pm 0,6 \\
1,9 \pm 0,7\end{array}$ & 2,096 & 0,413 & $0,0125 / 0,409$ \\
\hline
\end{tabular}

Con respecto a la correlación del IHO-S con el OHLA-S y las características sociodemográficas, observamos que hay correlación estadísticamente significativa con la edad del trabajador $(0,154)$ y con el puntaje del OHLA-S; sin embargo, esta se presentó de forma negativa $(-0,149)$, mostrando que los trabajadores con bajo puntaje en alfabetismo en salud oral tuvieron mejor higiene. En la correlación del IPC, se encontró correlación significativa con la edad del trabajador $(0,428)$, los años de escolaridad $(-0,208)$ y el OHLA-S $(-0,22)$ pero al ser negativa nos indica que a menor edad y menor alfabetismo en salud oral tienen mejor salud periodontal los trabajadores (Tabla IV).
Con respecto al análisis de regresión lineal simple, las variables independientes fueron analizadas por separado. En el IHO-S dos variables contribuyeron a la varianza con porcentajes significativos. Estas fueron el OHLA-S, explicando el 1,8\% de la varianza y la edad, explicando el 1,9\% de la varianza. Al analizar el valor de b, se observó que el OHLA-S presentó un b de $-0,02$, es decir, influye indirectamente en los puntajes de IHO-S. En el caso de la edad, este presentó un b de 0,14 , lo que mostrando la edad del trabajador influye positivamente en los puntajes de IHO$\mathrm{S}$ (Tabla V). En el caso del IPC se puede observar que dos variables son las que contribuyen a la varianza

Tabla IV. Correlación de pearson.

\begin{tabular}{|c|c|c|c|c|c|c|}
\hline & IHOS-S & $I P C$ & OHLA-S & Edad & $\begin{array}{l}\text { Ingreso } \\
\text { económico }\end{array}$ & $\begin{array}{l}\text { Años de } \\
\text { escolaridad }\end{array}$ \\
\hline & $r$ & $r$ & $r$ & $r$ & $r$ & $r$ \\
\hline IHO-S & - & $0,433^{* *}$ & $-0,149^{*}$ & $0,154^{*}$ & 0,013 & 0,66 \\
\hline IPC & $0,433^{* *}$ & - & $-0,229^{* *}$ & $0,428^{\star *}$ & $-0,056$ & $-0,208^{*}$ \\
\hline OHLA-S & $-0,149 *$ & $-0,229^{* *}$ & - & $-0,158^{*}$ & $0,234^{\star *}$ & $0,583^{\star *}$ \\
\hline
\end{tabular}

${ }^{*} p<0,05 \quad{ }^{* *} p<0,01$ 
GARCíA, M. D.; OTZEN, T.; DE LA FUENTE-HERNÁNDEZ, J. \& MANTEROLA, C. Higiene oral y salud periodontal en trabajadores agrícolas migrantes y su asociación con el alfabetismo funcional en salud oral. Int. J. Odontostomat., 15(1):159-165, 2021.

Tabla V. Regresión lineal simple variable dependiente IHO-Se IPC.

\begin{tabular}{|c|c|c|c|c|c|c|c|c|c|c|c|c|}
\hline \multirow{2}{*}{$\begin{array}{l}\begin{array}{l}\text { Variable } \\
\text { dependiente }\end{array} \\
\text { Variable } \\
\text { independiente }\end{array}$} & \multicolumn{6}{|c|}{ IHO-S } & \multicolumn{6}{|c|}{$I P C$} \\
\hline & $\mathrm{F}$ & $p$ & $\mathrm{R}_{2}$ & - & Error & $p$ & $\mathrm{~F}$ & $P$ & $\mathrm{R}^{2}$ & - & Error & $p$ \\
\hline OHLA-S & 4,701 & 0,031 & 0,018 & $-0,022$ & 0,010 & 0,031 & 11,407 & 0,001 & 0,048 & $-0,024$ & 0,007 & 0,001 \\
\hline Edad & 5,011 & 0,026 & 0,019 & 0,14 & 0,006 & 0,026 & 46,231 & $<0,001$ & 0,179 & 0,029 & 0,004 & $<0,001$ \\
\hline Ingreso & 0,034 & 0,854 & $-0,005$ & 0,00 & 0,002 & 0,854 & 0,422 & 0,422 & $-0,002$ & $-0,001$ & 0,002 & 0,422 \\
\hline $\begin{array}{l}\text { Años de } \\
\text { escolaridad }\end{array}$ & 0,904 & 0,343 & 0,000 & 0,020 & 0,021 & 0,343 & 9,300 & 0,003 & 0,039 & $-0,045$ & 0,015 & 0,003 \\
\hline Sexo & 2,33 & 0,128 & 0,006 & 0,21 & 0,13 & 0,128 & 0,706 & 0,402 & $-0,001$ & 0,085 & 0,101 & 0,402 \\
\hline $\begin{array}{l}\text { Visita al } \\
\text { dentista }\end{array}$ & 1,544 & 0,215 & 0,003 & 0,174 & 0,140 & 0,215 & 4,393 & 0,037 & 0,016 & $-0,211$ & 0,101 & 0.037 \\
\hline $\begin{array}{l}\text { Seguro de } \\
\text { salud }\end{array}$ & 1,850 & 0,175 & 0,004 & 0,209 & 0,153 & 0,175 & 0,213 & 0,645 & 0,004 & 0,051 & 0,112 & 0.645 \\
\hline
\end{tabular}

con porcentajes significativos. El OHLA-S, con un $\mathrm{R}^{2}$ de 0,048 , explicando el $4,8 \%$ de la varianza, la edad explicando el $17,9 \%$ de la varianza y los años escolares explicando el 3,9\% de la varianza. Al analizar el valor de $b$, se muestra que el OHLA-S tiene un $b$ de 0,024 , indicando que IPC influye disminuyendo el puntaje del OHLA-S (Tabla VI).

Para el análisis de regresión lineal múltiple se encontró que en el modelo del IHO-S las variables conjuntamente no explican las variaciones de salud oral de forma importante. Sin embargo al modelo del IPC le aportan varianza de forma conjunta la edad y OHLA$\mathrm{S}$, explicando el modelo un $22,0 \%$ de la varianza de IPC. Los valores de $b$ de ambas variables indican que al analizarlas en conjunto la edad sigue influyendo de forma indirecta a los puntajes de IPC pero más moderadamente por influencia de OHLA-S (Tabla VI)

Tabla VI. Regresión Lineal Múltiple.

\begin{tabular}{lccc}
\hline & $\mathrm{F}$ & $p$ & $\mathrm{R}^{2}$ \\
\hline Modelo & 27,256 & $<0,001$ & 0,202 \\
Variable independiente & - & Error & $P$ \\
OHLA-S & $-0,017$ & 0,007 & $<0,001$ \\
Edad & 0,027 & 0,004 & $<0,001$ \\
\hline
\end{tabular}

\section{DISCUSIÓN}

El estado de salud de un individuo se encuentra influenciado por diversas variables sociales, conductuales y económicas, mostrándose como determinantes en el proceso de salud y enfermedad de la boca (Medina-Solís et al., 2006); Sin embargo, para mantener un buen estado de salud oral, se requiere que la persona entienda y actúe sobre la información de salud que se le ha proporcionado de forma verbal o escrita (Lee et al., 2011).
Esta investigación es la primera que se realiza en población de habla hispana, en la cual se evalúa el alfabetismo funcional en salud oral y su asociación con el estado de salud oral. Considerando que nuestros sujetos fueron incluidos de un albergue de trabajadores agrícolas, los resultados pueden tener generalización limitada a otras poblaciones y deben ser interpretados con cuidado; ya que es poca la literatura que existe sobre la población de estudio. Nosotros encontramos un promedio del OHLA-S de 7,8 8 6,9 y aunque no se ha establecido el punto de corte para el OHLAS esta estimación es baja, considerando que el puntaje más alto del instrumento es 30 . Este promedio es justificable porque tenemos que el $73,6 \%$ de la población refiere no haber asistido a la escuela y encontramos diferencia estadísticamente significativa del puntaje del OHLA-S entre las personas que fueron o no a la escuela, de igual forma que Lee y Cols., encontraron diferencias entre los niveles de educación (Lee et al., 2012).

Las personas con mayor ingreso económico obtuvieron mayor puntaje en el OHLA-S, lo cual es similar a lo reportado en un estudio anterior, en el que se menciona que las puntuaciones del alfabetismo aumentan a mayor estado socioeconómico de las personas (Kanupuru et al.). Por otra parte, la población presentó buen estado de higiene oral y periodontal, sin presentar diferencias estadísticamente significativas por variables sociodemográficas. Al conocer que son una población desfavorable social y económicamente, esperaríamos encontrar una mala higiene y un mal estado periodontal, sin embargo es importante recordar que los conocimientos sobre salud oral pueden adquirirse de forma cotidiana, a lo largo de su existencia, como resultado de vivencias y experiencias (Dho, 2015). 
Al no encontrar diferencias estadísticamente significativas del IPC, IHO-S y el OHLA-S por tener seguro de salud, se sugiere que es porque el gobierno mexicano recientemente incorporó al sistema de asistencia médica a todas las personas que carecían de seguro médico y nuestra investigación no indagó en relación a si ellos hacen uso del servicio de salud en el área odontológica. Por otra parte, tampoco encontramos diferencias estadísticamente significativas del OHLAS, el IPC y el IHOS entre quienes han visitado al dentista, lo cual se debería reflejar porque el asistir al dentista aumentaría el alfabetismo en salud oral, así como, mejorar la higiene oral y el estado periodontal. Sin embargo, esta falta de diferencia se puede deber a que la información sobre salud actualmente está disponible para todos a través de diversos medios de comunicación, como son la televisión, radio, campañas de información y anuncios publicitarios (Pippi et al.).

Por otra parte, encontramos que el puntaje del OHLA-S influye poco en el estado de higiene oral, siendo estos resultados semejantes a los que fueron reportados en un estudio anterior (Lee et al., 2012). Por otra parte, la edad y el puntaje del OHLA-S, en conjunto influyen más sobre el estado periodontal.

Nuestros resultados difieren de los reportados en su mayoría por otros autores, sin embargo, es importante mencionar que el nivel de alfabetismo funcional en salud oral es sólo uno de los diversos factores que podrían influenciar en el estado de salud de las personas (Baskaradoss); siendo importante considerar otros aspectos como la cultural y el lenguaje. Además, cabe señalar que las condiciones estadísticas de la muestra no son las óptimas para realizar análisis de regresión lineal por lo que se sugiere a futuro considerar muestreos aleatorios de sujetos y tamaños de muestras mayores que permitan que las inferencias estadísticas aquí realizadas no presenten sesgos.

A modo de conclusión, podemos señalar que: Los trabajadores agrícolas migrantes presentaron un promedio en el índice de higiene oral considerada como buena higiene y el promedio del índice periodontal comunitario indica presencia de gingivitis. El nivel de alfabetismo funcional en salud es bajo; sin embargo, encontramos asociación del estado de higiene oral y el periodontal con el alfabetismo funcional; pero este último contribuye muy poco. Se sugiere continuar investigando a la población de estudio para conocer qué factores tienen mayor influencia en su estado de salud oral, así como mejorar su nivel de alfabetismo funcional en salud para garantizar su comprensión y entendimiento hacia las indicaciones de los profesionales en salud y mantener una buena salud oral.

GARCÍA, M. D.; OTZEN, T.; DE LA FUENTE-HERNÁNDEZ, J. \& MANTEROLA, C. Oral hygiene and periodontal health in migrant farm workers and its association with functional literacy in oral health. Int. J. Odontostomat., 15(1):159-165, 2021.

ABSTRACT: In Mexico, oral health disease is among the greatest health care demands in health services. These are evident in the higher vulnerability of migrant worker populations regarding major healthcare issues. Oral health literacy $(\mathrm{OHL})$ is the ability of individuals to obtain, process and understand information of basic health services. The aim of the study was to ascertain the state of oral hygiene and its relation with the level of oral health literacy of Mexican migrant farm workers. A cross-sectional study was carried out, with a sample size of 208 subjects selected for convenience and calculated by the expected OR. Subjects 18 years of age or older with signed informed consent were included. The variables studied were: Oral health literacy, simplified oral hygiene index (OHI-S) and Community Index of Periodontal (CPI). Descriptive and inferential analyses were performed. In this evaluation, $53.0 \%$ of the sample were men. The mean age was $30.7 \pm 10.8$ years. The average oral health literacy was $7.8 \pm 6.9$, the average hygiene index was $1.7 \pm 1.0$ and the community periodontal index was $2.0 \pm 0.7$. The multiple linear regression of the CPI shows that the age and OHLA-S scores account for $22.0 \%$ of the variance and the OHLA-S indirectly influences the IHO-S. This study reflects the importance of performing health interventions focused not only on oral health knowledge, but also considering the age of the individual and maintaining open future lines of research on this subject in different populations.

KEY WORDS: emigrants and immigrants, transients, migrants, health status, health status indicators, oral health, oral hygiene, oral hygiene index, periodontal index.

\section{REFERENCIAS BIBLIOGRÁFICAS}

Baskaradoss, J. K. The association between oral health literacy and missed dental appointments. J. Am. Dent. Assoc., 147(11):86774,2016

Blizniuk, A.; Ueno, M.; Zaitsu, T. \& Kawaguchi, Y. Association of oral health literacy with oral health behaviour and oral health status in Belarus. Community Dent. Health, 32(3):148-52, 2015.

Burgette, J. M.; Lee, J. Y.; Baker, A. D. \& Vann Jr., W. F. Is dental utilization associated with oral health literacy? J. Dent. Res., 95(2):160-6, 2016. 
GARCíA, M. D.; OTZEN, T.; DE LA FUENTE-HERNÁNDEZ, J. \& MANTEROLA, C. Higiene oral y salud periodontal en trabajadores agrícolas migrantes y su asociación con el alfabetismo funcional en salud oral. Int. J. Odontostomat., 15(1):159-165, 2021.

Dho, M. S. Conocimientos de salud bucodental en relación con el nivel socioeconómico en adultos de la ciudad de Corrientes, Argentina. Rev. Fac. Nac. Salud Pública, 33(3):361-9, 2015.

Encuesta Nacional de Jornaleros Agrícolas (ENJO), 2009. Disponible en: http://www.cipet.gob.mx/jornaleros

Gamboa Montejano, C. \& Gutiérrez Sánchez, M. Jornaleros Agrícolas en México. Antecedentes, Políticas Públicas, Tratados Internacionales, Causas y Efectos del Problema, Iniciativas y Opiniones Especializadas. Ciudad de México, Cámara de Diputados, Dirección de Servicios de Investigación y Análisis, Subdirección de Análisis de Política Interior, 2015. Disponible en: http://www.diputados.gob.mx/sedia/sia/spi/SAPI-ISS-7815.pdf

Haridas, R.; Supreetha, S.; Ajagannanavar, S. L.; Tikare, S.; Maliyil, M. J. \& Kalappa, A. A. Oral health literacy and oral health status among adults attending Dental College Hospital in India. J. Int. Oral Health, 6(6):61-6, 2014.

Juárez-Sánchez, J. P. Migración indígena hacia espacios agrícolas marginados de México. Un caso para contar. Agric. Soc. Desarro., 12(1):87-105, 2015.

Kanupuru, K. K.; Fareed, N. \& Sudhir, K. M. Relationship between oral health literacy and oral health status among college students. Oral Health Prev. Dent., 13(4):323-30, 2015.

Lee, H. Y.; Rhee, T. G.; Kim, N. K. \& Ahluwalia, J. S. Health literacy as a social determinant of health in Asian American immigrants: findings from a population-based survey in California. J. Gen. Intern. Med., 30(8):1118-24, 2015.

Lee, J. Y.; Divaris, K.; Baker, A. D.; Rozier, R. G. \& Vann Jr., W. F. The relationship of oral health literacy and self-efficacy with oral health status and dental neglect. Am. J. Public Health, 102(5):923-9, 2012.

Lee, J. Y.; Divaris, K.; Baker, A. D.; Rozier, R. G.; Lee, S. Y. D. \& Vann Jr., W. F. Oral health literacy levels among a low-income WIC population. J. Public Health Dent., 71(2):152-60, 2011.

Lee, J.; Stucky, B.; Rozier, G.; Lee, S. Y. \& Zeldin, L. P. Oral Health Literacy Assessment: development of an oral health literacy instrument for Spanish speakers. J. Public Health Dent., 73(1):18, 2013.

Manterola, C. \& Astudillo, P. Checklist for Reporting of Descriptive Observational Studies. MINCIR Initiative. Int. J. Morphol., 31(1):115-20, 2013.

Medina-Solís, C. E.; Segovia-Villanueva, A.; Estrella-Rodríguez, R.; Maupomé, G.; Ávila-Burgos, L. \& Pérez-Nuñez, R. Asociación del nivel socioeconómico con la higiene bucal en preescolares bajo el programa de odontología preventiva del IMSS en Campeche. Gac. Med. Mex., 142(5):363-8, 2006.

Ospina, D.; Herrera, Y.; Betancur, J.; Agudelo, H. B. \& Posada López, A. Higiene bucal en la población de San Francisco Antioquia y sus factores relacionados. Rev. Nac. Odontol., 12(22):23-30, 2016.

Pippi, R.; Bagnato, F. \& Ottolenghi, L. Oral Health Literacy: How much Italian people know about the dental hygienist. J. Clin. Exp. Dent., 9(1):e13-20, 2017.

SEDESOL. Tendencias recientes de la migración interna de los jornaleros agrícolas. Ciudad de México, Gobierno de México, Secretaria de Desarrollo Social, 2006.
Dirección para correspondencia:

Dra. Tamara Otzen

Profesora Centro de Excelencia en Estudios Morfológicos y

Quirúrgicos (CEMyQ)

Doctorado en Ciencias Médicas

Universidad de La Frontera

Temuco

CHILE

ORCID: 0000-0001-6014-1241

E-mail: tamara.otzen@ufrontera.cl 